\title{
Malignant lymphoma occurring simultaneously in the urinary bladder wall and bilateral renal parenchyma: A case report
}

\author{
JIE MIN*, HAO GENG* ${ }^{*}$, DEXIN YU, TAO ZHANG and ZHIQIANG ZHANG \\ Department of Urology, The Second Hospital of Anhui Medical University, Hefei, Anhui 230022, P.R. China
}

Received September 16, 2014; Accepted June 2, 2015

DOI: $10.3892 / 01.2015 .3439$

\begin{abstract}
A 67-year-old man was admitted to hospital with acid regurgitation, acute right lower abdominal pain and mild fever. Multiple suspicious mass lesions were detected in the patient's kidneys, the right side wall of his bladder and the right lower ureter, using CT urography and PET-CT. He underwent palliative surgery of the bladder and percutaneous renal biopsy. Postoperative histopathological examination and immunohistochemical staining indicated that the tumor was consistent with diffuse large B-cell lymphoma (DLBCL). Although DLBCL is by far the most common type of non-Hodgkin's lymphoma, it is rare for DLBCL to simultaneously involve the kidneys, bladder and the lower ureteric tract.
\end{abstract}

\section{Introduction}

Non-Hodgkin's lymphoma (NHL) arises from B or T lymphocytes. Extranodal lymphoma originating in solid organs, accounts for one-third of all cases of NHL, and is predominantly associated with breast, kidney, colon and rectal carcinoma (1). By contrast, the majority of bladder tumors are derived from the epithelium. Non-epithelial tumors of the bladder are extremely rare (2). However, secondary bladder involvement occurs in $10-20 \%$ of cases of terminal NHL. Since the presentation of diffuse large B-cell lymphoma (DLBCL) is nonspecific, the diagnosis is often only made following histological examination. Tumor cells are strongly positive for CD79a, CD20 and the bcl 2 protein. The proliferative fraction of cells, as determined by Ki-67 staining, is $~ 70 \%$, compared with $<25 \%$ of normal urothelial cells of the bladder (3). Frequently, DLBCL tumors are negative for cytokeratins and vimentin. These immunohistochemical characteristics allow for the accurate diagnosis of DLBCL following tumor biopsy $(2,4)$.

Correspondence to: Dr Dexin Yu, Department of Urology, The Second Hospital of Anhui Medical University, 678 Furong Road, Hefei, Anhui 230022, P.R. China

E-mail:79478900@qq.com

${ }^{*}$ Contributed equally

Key words: malignant lymphoma, bladder tumor, renal tumor
The current report presents an unusual case of DLBCL, involving the kidney, bladder and the lower ureter, which was detected by imaging and confirmed by histopathological examination. The clinical findings and experience with this patient suggest an appropriate and practical course of action when dealing with similar cases. Written informed consent was obtained from the patient for inclusion in the present study.

\section{Case report}

A 67-year-old man initially presented with acid regurgitation and mild fever lasting for $>20$ days, in conjunction with acute constant pain in the right lower quadrant of his abdomen for 4-5 days and $5 \mathrm{~kg}$ of weight loss over the past 3 months. The patient had a history of chronic appendicitis, digestive disorders, including reflux esophagitis and chronic superficial gastritis, as well as hepatobiliary diseases. Physical examination revealed tenderness in McBurney's point. A series of routine laboratory tests demonstrated a normal white blood cell count $\left(4.75 \times 10^{9}\right.$ cells/l) and a hemoglobin concentration of $99 \mathrm{~g} / 1$. Urinary analysis revealed occult blood 1+ and a red blood cell count of 9.6 cells $/ \mu 1$. Procalcitonin was $0.056 \mathrm{ng} / \mathrm{ml}$ (normal range, $0-0.046 \mathrm{ng} / \mathrm{ml}$ ) and interleukin-6 was $22 \mathrm{pg} / \mathrm{ml}$ (normal range, $0-7 \mathrm{pg} . / \mathrm{ml}$ ), indicating that the patient did not have an infection. Blood urea nitrogen and serum creatinine were $4.65 \mathrm{mmol} / 1$ (normal range, 3.2-7.1 mmol/l) and $114 \mu \mathrm{mol} / 1$ (normal range, 53-106 $\mu \mathrm{mol} / \mathrm{l}$ ), respectively. Gastroscopy demonstrated reflux esophagitis and chronic superficial gastritis. In order to investigate the patient's fever and persistent abdominal pain, abdominal and pelvic CT, and CT urography (CTU) scans were performed, which revealed multiple masses in both kidneys, the right lateral wall of the bladder and the right lower ureter, in addition to hydronephrosis (Fig. 1). PET-CT demonstrated hypermetabolic regions in the same locations. The left submandibular and intertrochanteric area also exhibited an increase in fluorodeoxyglucose metabolism (Fig. 2).

Although the $\mathrm{CT}$ scan indicated bilateral renal pelvic masses, with right lower ureter and bladder invasion, this was suspected to be a systemic malignant tumor rather than an advanced-stage classic urinary carcinoma. Due to the poor renal function and the patient's pain as a result of the obstruction in the right lower ureter, diagnostic and palliative surgery was performed. The lower mass was completely resected by partial cystectomy and ureteric re-implantation. 


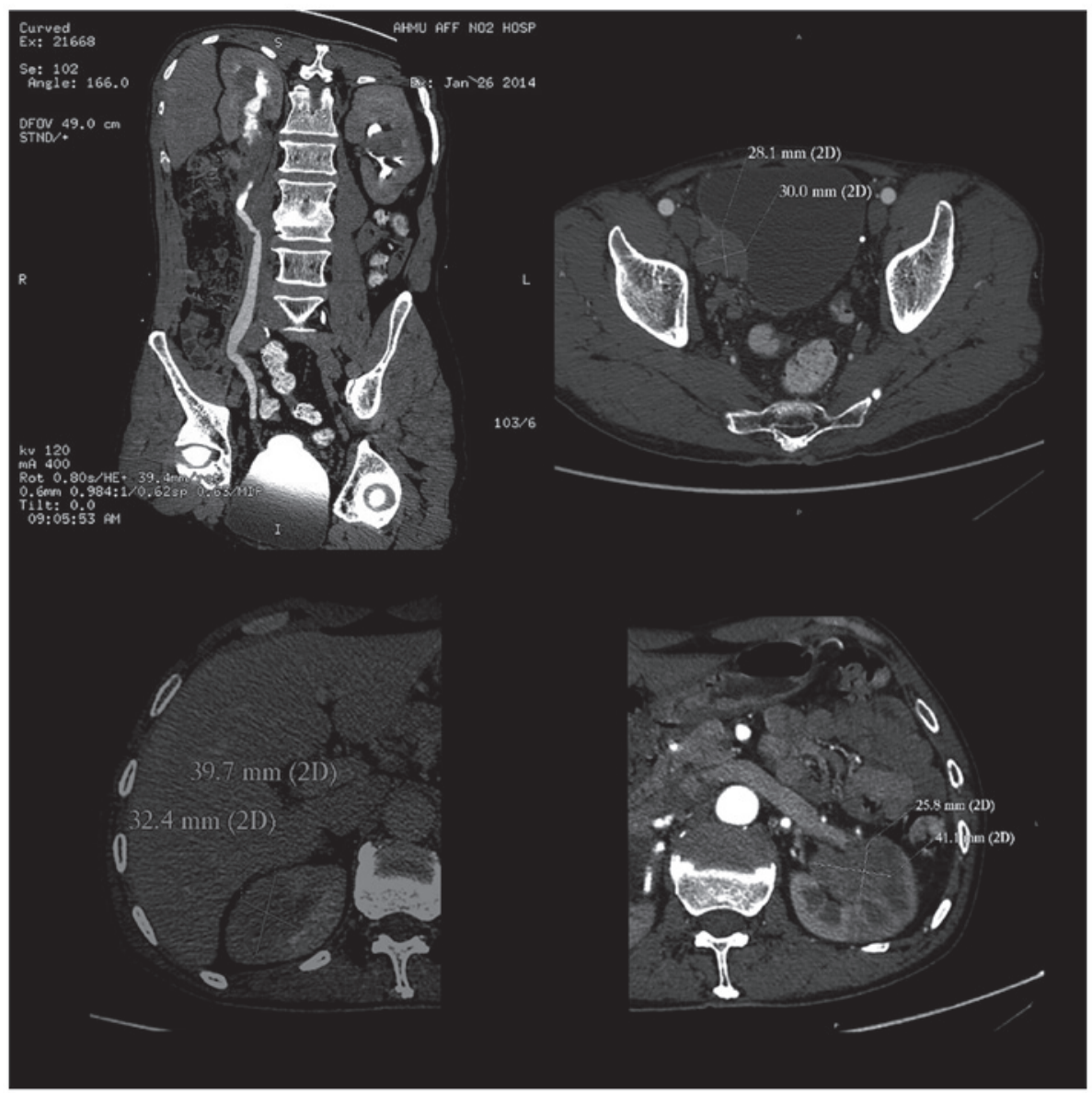

Figure 1. CT urography demonstrated multiple masses in both kidneys, the right wall of the bladder and the right lower ureter, in addition to hydronephrosis.

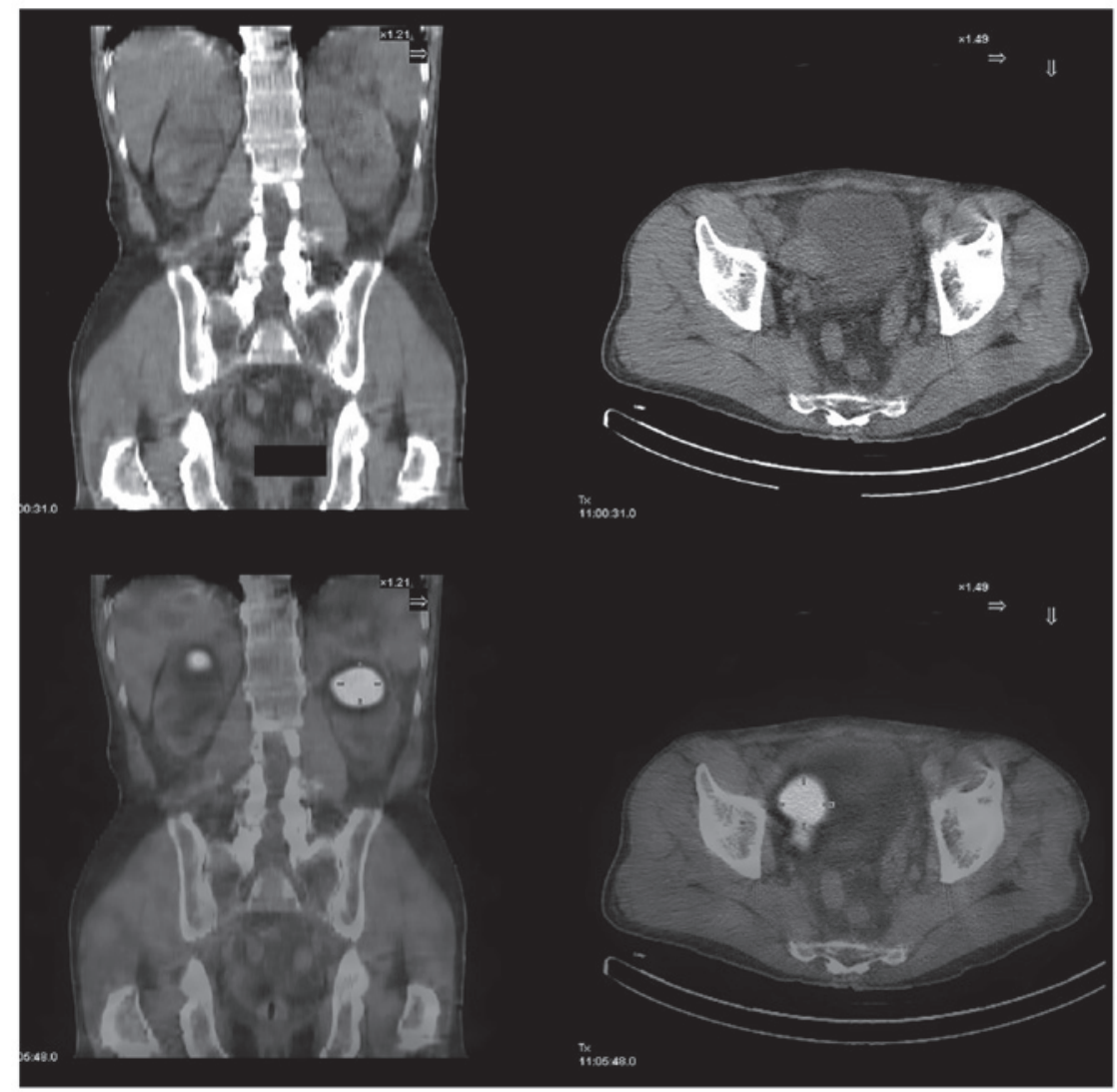

Figure 2. PET-CT demonstrated hypermetabolic regions in the same locations as the CT images. 
A

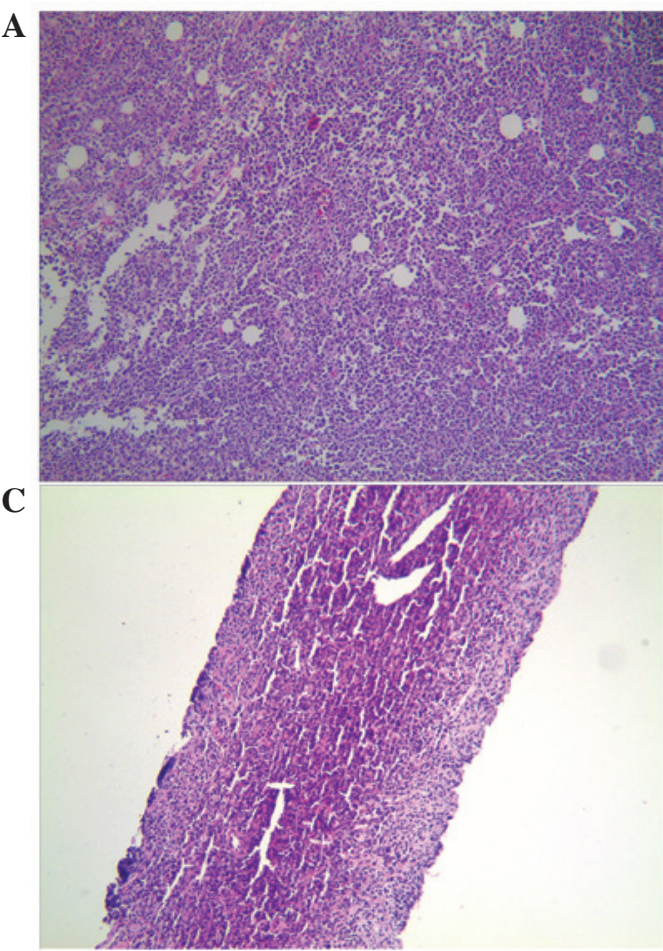

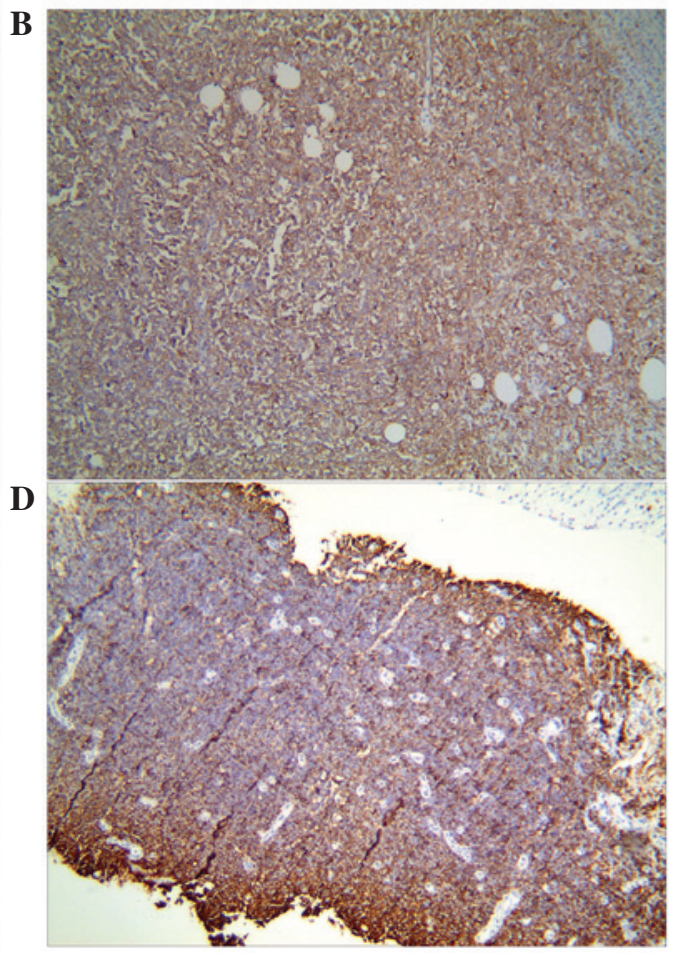

Figure 3. Hematoxylin and eosin, and immunohistochemistry staining of the tissue from the bladder mass specimen (A) and (B) and the percutaneous renal biopsy (C) and (D).

Histopathological examination of the mass suggested a small-cell malignancy, invading the bladder floor. Immunohistochemistry staining indicated the presence of the leukocyte common antigen and the B-cell Marker, CD20, and was negative for cytokeratin. These findings were consistent with a diagnosis of DLBCL (Fig. 3A and B). It was suspected that the kidney masses may also be part of the lymphoma, and therefore a percutaneous renal biopsy was conducted. Pathological examination of the biopsy, combined with clinical data, supported the diagnosis of a small-cell malignancy, which was most likely to be DLBCL (Fig. 3C and D). Consequently, the patient was transferred to the Department of Hematology for further diagnosis and treatment. The patient was followed up in the medical department and bone marrow biopsy demonstrated active proliferation in bone marrow cells, with no obvious abnormalities on immunophenotyping. The final diagnosis was reported as DLBCL, originating from bone marrow, and involving bladder and kidney. To date, the patient has received his first cycle of R-CHOP (rituximab, cyclophosphamide, doxorubicin, vincristin and prednisone) chemotherapy.

\section{Discussion}

DLBCL is by far the most common type of NHL lymphoma and accounts for 30,000 new cases per year in the United States (5). However, DLBCL simultaneously involving the kidney, the bladder and the lower ureter, as reported here, is very rare in clinical practice.

Bladder lymphoma may present as the first sign of extranodal lymphoma and is often asymptomatic, although symptoms may include hematuria, lower urinary tract symptoms, flank pain and pelvic pain (6). The cystoscopic appearance of lymphoma may be mistaken for urothelial carcinoma, although the tumors are often described as nodular and may be covered by normal urothelium (6).

Lymphoma of the urinary bladder may represent either primary lymphoma or secondary involvement by systemic lymphoma, which is more common. Secondary involvement of the urinary bladder occurs in 10-25\% of advanced-stage leukemias/lymphomas (7). Primary bladder lymphoma is most frequently of the mucosa-associated lymphatic tissue (MALT) type, while secondary lymphoma generally arises from DLBCL, as in the present case. The 'MALT concept', which was first proposed by Isaacson and Wright in 1983 (8) and first reported as a case arising in the bladder in 1990 (9), suggests a mechanism by which malignant lymphomas may develop in the bladder and in other sites that normally possess no lymphoid tissue, following a period of chronic inflammation. It has been reported that DLBCL may also arise following transformation of MALT.

Immunohistochemistry is the most useful examination to distinguish between the two types of lymphoma (MALT and DLBCL). However, transurethral resection is often required in order to obtain samples for pathological diagnosis. In the present case, a partial cystectomy and resection of the lower ureter were performed in order to relieve the symptoms of obstruction and to obtain samples for histopathological examination. Complete resection of the bladder tumor or even radical cystectomy are considered unnecessary, as the tumor often responds favorably to chemotherapy, of which the CHOP regimen is the most widely used protocol $(10,11)$. The addition of rituximab in CD20-positive cases, is recommended to be standard practice, due to the therapeutic benefit provided 
by this monoclonal antibody in the treatment of NHL (2). Response rates of isolated primary bladder lymphoma to chemotherapy have been reported to be favorable, while non-localized and secondary (recurrent) lymphomas have a poorer prognosis. Kempton et al (12) reported a longer median survival for primary bladder lymphoma compared with systemic lymphoma (NHL) involving only the urinary bladder. Secondary lymphoma in the urinary bladder is associated with widely disseminated disease and a poor prognosis (7).

Extranodal lymphoma originating in solid organs, accounts for one-third of all cases of NHL, and often involves the kidneys (13). By contrast, primary renal lymphoma (PRL) is a rare disease, the existence of which is still debated, since the kidney is devoid of lymphatic tissue (14-16). It has been proposed that PRL may originate in the lymph nodes of the renal sinus or in the lymphatic network of the renal capsule, and that it forms cords of cells that penetrate the renal parenchyma (16). There is a consensus that the criteria for PRL should include: Renal failure as the initial presentation; enlargement of the kidneys without obstruction, and also without other organ or nodal involvement; absence of other causes of renal failure; a diagnosis made by renal biopsy; and rapid improvement of renal function following chemotherapy.

Renal lymphoma is often ill-defined on CT imaging, with no capsule, a homogeneous appearance and slightly higher than normal density renal parenchyma on unenhanced scanning. The lesions exhibit a greater enhancement than normal renal parenchyma, following the intravenous administration of contrast. On MR imaging, the lesions are hypointense on T1-weighted images and hypointense on T2-weighted images. The tumor frequently infiltrates the whole renal parenchyma and sinuses, causing an enlarged kidney or encasement of the renal vessels.

However, renal lymphoma has a wide variety of manifestations on imaging. Therefore, the diagnosis may be difficult, and this may be more complicated when attempting to differentiate between PRL and secondary renal lymphoma. In addition, diagnoses are occasionally established unexpectedly following radical nephrectomy, since the tumors are initially assumed to be renal cell carcinoma (17).

Takahashi et al (18) reported a retrospective study, evaluating the prognostic effect of various extranodal sites in 1221 patients with extranodal DLBCL. In this series, all patients were positive for CD20 and received treatment with R-CHOP therapy. The median observation period in surviving patients was 47.9 months. The 5-year overall survival rate of the total cohort was $75.0 \%$. Notably, patients with $>1$ extranodal site had a significantly poorer prognosis than those with $\leq 1$ extranodal sites (5-year overall survival, 54.2 vs. $80.2 \%$; $\mathrm{P}<0.001)$. Furthermore, extranodal kidney involvement was associated with significantly decreased overall survival $(\mathrm{P}<0.05)$. These findings indicate that extranodal involvement affects the prognosis of patients undergoing R-CHOP therapy for DLBCL.

Despite the high frequency of renal involvement in DLBCL, simultaneous involvement of both kidneys, the urinary bladder and the lower ureteric tract, is very rare. The possibility of extranodal involvement in systemic malignant lymphoma should be taken into consideration when formulating a differential diagnosis for complicated urinary tumors. Surgeons should undertake a full assessment of the patient, including reviewing imaging and examination reports, and liaise with colleague from medicine, imaging and pathology, in order to deliver the optimum treatment and to avoid unnecessary surgery intervention.

\section{References}

1. Cabuk D, Gullu YT, Basyigit I, Acikgoz O, Uygun K, Yildiz K and Yildiz F: Multifocal extranodal involvement of diffuse largeb-cell lymphoma. Case Rep Pulmonol 2013: 794642, 2013.

2. Mahfoud T, Tanz R, Mesmoudi M, Khmamouche MR, El Khannoussi B, Ichou M and Errihani H: Primary non-Hodgkin's lymphoma of the bladder: Case report and literature review. Pan Afr Med J 15: 136. 2013.

3. Ding W, Gou Y, Sun C, Xia G, Wang H, Chen Z, Tan J, Xu K and Qiang D: Ki-67 is an independent indicator in non-muscle invasive bladder cancer (NMIBC); combination of EORTC risk scores and Ki-67 expression could improve the risk stratification of NMIBC. Urol Oncol 32: 42.e13-42. e19, 2014.

4. Venyo AK: Lymphoma of the urinary bladder. Adv Uro 2014: 327917, 2014

5. Lohr JG, Stojanov P, Lawrence MS, Auclair D, Chapuy B, Sougnez C, Cruz-Gordillo P, Knoechel B, Asmann YW, Slager SL, et al: Discovery and prioritization of somatic mutations in diffuse large B-cell lymphoma (DLBCL) by whole-exome sequencing. Proc Natl Acad Sci USA 109: 3879-3884, 2012.

6. Johnson MH, Nepple KG and Humphrey PA: Bladder lymphoma. J Urol 188: 269-270, 2012.

7. Levy G: Bladder other tumors Lymphoma (primary), PathologyOutlines.com: http://www.pathologyoutlines. com/topic/bladderlymphoma.html, 2011.

8. Isaacson $\mathrm{P}$ and Wright DH: Malignant lymphoma of mucosa-associated lymphoid tissue. A distinctive type of B-cell lymphoma. Cancer 52: 1410-1416, 1983.

9. Kuhara H, Tamura Z, Suchi T, Hattori R and Kinukawa T: Primary malignant lymphoma of the urinary bladder. A case report. Acta Pathol Jpn 40: 764-769, 1990.

10. Antunes AA, Nesrallah LJ and Srougi M: Non-Hodgkin lymphoma of the bladder. Int Braz J Urol 30: 499-501, 2004.

11. Leite KRM, Bruschini H and Camara-Lopes LH: Primary lymphoma of the bladder. Int Braz J Urol 30: 37-39, 2004.

12. Kempton CL, Kurtin PJ, Inwards DJ, Wollan P and Bostwick DG: Malignant lymphoma of the bladder: Evidence from 36 cases that low-grade lymphoma of the MALT-type is the most common primary bladder lymphoma. Am J Surg Pathol 21: 1324-1333, 1997.

13. Cabuk D, Gullu YT, Basyigit I, Acikgoz O, Uygun K, Yildiz K and Yildiz F: Multifocal extranodal involvement of diffuse large B-Cell lymphoma. Case Rep Pulmonol 2013: 794642, 2013.

14. Pahwa M, Gupta N, Tyagi V and Chadha S: Primary renal lymphoma: Is prognosis really that bad? Saudi J Kidney Dis Transpl 24: 816-817, 2013.

15. Hart S, Ellimoottil C, Shafer D, Mehta V and Turk TM: A case of primary renal lymphoma. Urology 80: 763-765, 2012.

16. Torrecilla García-Ripoll JR, Pascual Samaniego M, Martín Blanco S, Rivera Ferro J, Peral Martínez JI and Fernández del Busto E: Primary renal lymphoma. Actas Urol Esp 27: 555-558, 2003 (In Spanish).

17. Valarmathi K, Jamila A, Ravi S, Selvambigai and Muthulatha: A rare case of renal tumour. J Clin Diagn Res 7: 2006-2007, 2013.

18. Takahashi H, Tomita N, Yokoyama M, Tsunoda S, Yano T, Murayama K, Hashimoto C, Tamura K, Sato K and Ishigatsubo Y: Prognostic impact of extranodal involvement in diffuse large B-cell lymphoma in the rituximab era. Cancer 118: 4166-4172, 2012. 